\title{
Evaluación ecográfica de la función diafragmática en el paciente crítico
}

Charco Roca LM, Simón Polo E

Hospital General Universitario de Albacete.

\section{Resumen}

La ecografía ha surgido como una técnica no invasiva que se puede utilizar en la evaluación estructural y funcional del diafragma. Los ultrasonidos permiten observar el movimiento diafragmático en una variedad de condiciones normales y patológicas. Exponemos cuatro casos clínicos en los que la evaluación de la disfunción diafragmática por ecografía permitió hacer un diagnóstico del origen de la insuficiencia respiratoria de forma rápida y no invasiva. La ecografía del diafragma tiene su aplicación principalmente en pacientes en cuidados intensivos y en un futuro podría valorarse como modalidad de elección para la evaluación de la parálisis del diafragma con potencial aplicación clínica para el destete de la ventilación mecánica y diagnóstico diferencial de las causas de insuficiencia respiratoria.

\section{Introducción}

La ecografía ha surgido como una técnica no invasiva que se puede utilizar en la evaluación estructural y funcional del diafragma. Los ultrasonidos permiten observar el movimiento diafragmático en una variedad de condiciones normales y patológicas. Exponemos cuatro casos clínicos en los que la evaluación de la disfunción diafragmática por ecografía permitió hacer un diagnóstico del origen de la insuficiencia respiratoria de forma rápida y no invasiva. La ecografía del diafragma tiene su aplicación principalmente en pacientes en cuidados intensivos $y$ en un futuro podría valorarse como modalidad de elección para la evaluación de la parálisis del diafragma con potencial aplicación clínica para el destete de la ventilación mecánica y diagnóstico diferencial de las causas de insuficiencia respiratoria.

Puedes consultar las aplicaciones que recomendamos en ediciones anteriores. a ecografía en las unidades de cuidados críticos está imponiéndose en los últimos años como herramienta de diagnóstico rápido y monitorización en muy variadas patologías. Un mejor entendimiento de la fisiopatología del paciente crítico y la accesibilidad actual a los sistemas portátiles de ultrasonidos está revolucionando la exploración a pie de cama.

La ecografía ha surgido como una técnica no invasiva que se puede utilizar en la evaluación estructural y funcional del diafragma. Los ultrasonidos permiten observar el movimiento diafragmático en una variedad de condiciones normales y patológicas; puede evaluar las características del movimiento diafragmático, como la amplitud, la fuerza y la velocidad de contracción, los patrones especiales de movimiento y los cambios en el grosor del diafragma durante la inspiración.

Estos parámetros diafragmáticos ecográficos pueden proporcionar 
información valiosa en la evaluación y el seguimiento de pacientes con debilidad diafragmática o parálisis, y pueden ayudar potencialmente a comprender la disfunción pulmonar causante de una insuficiencia respiratoria.

Las mediciones por ultrasonido del espesor del diafragma son factibles $\mathrm{y}$ altamente reproducibles. En volúmenes inspiratorios clínicamente relevantes, el engrosamiento del diafragma refleja la contracción muscular y no la inflación pasiva. Esta técnica puede emplearse confiablemente para controlar el espesor, la actividad y la función del diafragma durante la ventilación espontánea y mecánicamente asistida. Este artículo revisa a propósito de cuatro casos clínicos la técnica y las aplicaciones clínicas de la ecografía en la evaluación de la función diafragmática en pacientes con insuficiencia respiratoria ingresados en una unidad de cuidados intensivos por diversos motivos.

\section{Descripción de casos clínicos}

\section{Caso 1: Parálisis diafragmática} secundaria a lesión medular cervical. Varón de 65 años sin antecedentes médico-quirúrgicos relevantes, que ingresó en la unidad de cuidados intensivos con el diagnostico de politraumatizado tras caída accidental desde tres metros de altura. El paciente presentaba un traumatismo craneoencefálico leve, traumatismo facial y traumatismo de raquis con fractura-luxación de $\mathrm{C} 6-\mathrm{C} 7 \mathrm{y}$ fractura de la lámina izquierda y apófisis espinosa de C4. Se indicó una resonancia magnética cervical urgente que mostró la presencia de subluxación C6-C7, con protrusión discal y presencia de hematoma epidural anterior a la altura de C6. Las imágenes mostraban una mínima deformidad medular con pequeñas zonas parcheadas de mielopatía y con otra zona más marcada de mielopatía por detrás de C3C4. Con los hallazgos radiológicos y el diagnostico de lesión medular incompleta ASIA C con la exploración clínica el paciente fue intervenido de forma urgente realizándose discectomía y fijación de cuerpos vertebrales C6-C7 con placa por vía cervical anterior. Tras la intervención se procedió a despertar al paciente observando adecuadas condiciones neurológicas y mostrando buena dinámica respiratoria $\mathrm{y}$ volúmenes pulmonares con ventilación mecánica en modo CPAP $(+6$ CmH2O). Como prueba de extubación se puso en tubo en $\mathrm{T}$ y realizamos ecografía en plano subcostal observando adecuada excursión diafragmática. Al considerar segura la extubación esta fue realizada. El paciente permaneció en observación clínica los minutos posteriores y empezó a observarse empleo de la musculatura intercostal y abdominal accesorias en los ciclos respiratorios, asimismo refería sensación disneica. No se observó desaturación en el monitor de pulsioximetría. En este momento se realizó una ecografía diafragmática en modo $M$ que mostraba ausencia de movilidad diafragmática significativa bilateral observando una fracción de acortamiento diafragmática nula. La radiografía de tórax no mostraba hallazgos significativos dado que la paresia diafragmática era bilateral y la inspiración se realizaba a consta de esfuerzo de musculatura accesoria (figura 1). Con estos signos clínicos y conociendo que el paciente tenía lesión medular cervical alta se decidió realizar traqueotomía quirúrgica ante la previsión de agotamiento respiratorio del paciente dada la afectación de la función diafragmática de origen neuromuscular. El paciente posteriormente permaneció con soporte ventilatorio según sus necesidades y tras ser intervenido de las fracturas panfaciales fue derivado a un centro de 
referencia especializado en lesión medular aguda para continuación de cuidados.

\section{Caso 2: Aquinesia diafragmática por denervación traumática.}

Varón de 53 años ingresado en la unidad de cuidados intensivos tras traumatismo por accidente de motocicleta. El balance lesional de ingreso fue de traumatismo craneoencefálico severo, traumatismo facial, traumatismo torácico derecho con rotura de aorta ascendente contenida, neumotórax, contusión pulmonar y fracturas costales múltiples, traumatismo locomotor con fractura de cuello femoral izquierdo, traumatismo de raquis con fractura de apófisis transversas derechas C7, D2, D4, y todas las lumbares y aumento espacio epidural posterior $\mathrm{C} 2-\mathrm{C} 4$ en relación con hematoma cervical. El paciente presentó disfunción multiorgánica $\mathrm{y}$ posterior evolución respiratoria tórpida, en las radiografías se observaba imagen de consolidación pulmonar (contusión / sobreinfección) y derrame pleural izquierdo persistente con elevación hemidiafragmática derecha (figura 1).

Tras la estabilización de las múltiples disfunciones orgánicas se procedió a retirada de sedación y destete de la ventilación mecánica siendo este muy dificultoso. Procedimos a evaluar la función diafragmática derecha observando ausencia de excursión diafragmática y en modo $\mathrm{M}$ ausencia de contracción traducida en fracción de acortamiento nula (figura 2). Se solicitó un estudio neurofisiológico que concluyó que presentaba una plexopatía braquial derecha severa, global-difusa, con mayor probabilidad completa. Asimismo con técnica electromiográfica mediante guía ecográfica se apreció denervación severa del hemidiafragma derecho compatible con neuropatía frénica de gran severidad. El destete respiratorio de este paciente precisó de descensos progresivos muy lentos del soporte ventilatorio y mucho apoyo de fisioterapia respiratoria.

\section{Caso 3: Disfunción de marcapasos diafragmático.}

Varón de 63 años que a consecuencia de un traumatismo ingresó en la unidad de cuidados intensivos con los diagnósticos de traumatismo craneoencefálico leve, traumatismo facial $y$ traumatismo vertebral con lesión medular a nivel de C2, ASIA A, con shock neurogénico, tetraplejia e insuficiencia respiratoria. Tras fijación quirúrgica de $\mathrm{C} 2$ a $\mathrm{C} 5$ y estabilización clínica fue traslado a un centro de referencia de manejo de lesionados medulares para continuación de cuidados. Por insuficiencia respiratoria de origen neuromuscular se le implanta marcapasos frénico que permite ventilación las 24 horas del día no precisando de ventilación invasiva domiciliaria. Tras tres meses del implante del marcapasos diafragmático acude a urgencias con signos de infección respiratoria $\mathrm{y}$ datos de insuficiencia respiratoria aguda hipercápnica. En la exploración radiológica se observa elevación marcada de hemidiafragma derecho (Figura 1). La exploración ecográfica permitió el diagnóstico rápido en el servicio de urgencias de ausencia de movilidad de hemidiafragma derecho que justifica el deterioro respiratorio del paciente. Tras ser diagnosticado de disfunción de marcapasos diafragmático es ingresado en la unidad de cuidados intensivos para soporte ventilatorio. Entre las complicaciones derivadas de estos dispositivos se incluye la posibilidad de fallo del marcapasos que se manifiesta con signos de insuficiencia respiratoria con necesidad de soporte ventilatorio y clínica de sobreinfección por mala movilización de secreciones. El paciente fue trasladado al centro de referencia de 
manejo de lesión medular para revisión y ajuste del dispositivo.

\section{Caso 4: Paresia diafragmática unilateral tras hepatectomía derecha.}

Mujer de 72 años ingresada en la unidad de cuidados intensivos para vigilancia postoperatoria tras hepatectomía derecha por quiste simple recidivado. Entre sus antecedentes destacaban hipertensión arterial, exfumadora de 1 año previo, con bronquiectasias y varios ingresos por sobreinfecciones respiratorias y diagnosticada de probable obstrucción crónica al flujo aéreo. Fue intervenida de quiste hepático abscesificado hacía un año y ya entonces presentó un postoperatorio tórpido con insuficiencia respiratoria y necesidad de traqueotomía en la fase de destete de la ventilación mecánica. En el primer día de postoperatorio actual presenta insuficiencia respiratoria y en la radiografía de tórax de observa elevación diafragmática derecha con aire subfrénico y hemitórax derecho con patrón intersticial difuso, sin claros infiltrados (Figura 1). Realizamos ecografía diafragmática derecha en la que se muestra ausencia de contracción diafragmática y presencia de derrame pleural basal leve. La paciente realiza fisioterapia respiratoria intensiva y tras tres días se observó recuperación de la movilidad y contracción diafragmática explorada por ecografía. La sospecha diagnostica de la causa de disfunción diafragmática transitoria fue de paresia irritativa por contigüidad con el sitio quirúrgico.

\section{Discusión}

El diafragma es el principal músculo respiratorio. La disfunción diafragmática puede ser causada por afecciones que afectan directamente al diafragma, como traumatismo, cirugía cardiotorácica, patología torácica o abdominal adyacente (p. Ej., Atelectasia pulmonar basal, neumonía o tumores), masas abdominales superiores, fluido pleural o abdominal extenso y distrofias musculares. El movimiento del diafragma también puede verse afectado por enfermedades del sistema nervioso central, lesión del nervio frénico en su trayecto en el cuello y el tórax, enfermedad de la neurona motora $y$ enfermedades de la unión neuromuscular (1).

La disfunción diafragmática tiene una alta incidencia en el paciente crítico y es una causa poco reconocida de insuficiencia respiratoria $y$ destete prolongado de la ventilación mecánica. El motivo de que esta entidad se encuentre infradiagnosticada reside en su presentación variada y a menudo inespecífica. Los hallazgos clínicos incluyen disnea inexplicable, especialmente en posición supina, dificultad para destetar de la ventilación mecánica, elevación del diafragma en las radiografías de tórax, dificultad respiratoria inexplicable, patrón de respiración asimétrica, movimiento paradójico abdominal, neumonía recurrente o colapso pulmonar unilateral recurrente.

El diagnóstico precoz de la disfunción diafragmática es importante, porque la parálisis diafragmática puede ser susceptible a estrategias terapéuticas y puede requerir un soporte ventilatorio adaptado y prolongado. Por lo tanto, la necesidad de evaluar la función del diafragma surge en muchas situaciones clínicas. En este artículo hemos expuesto diferentes casos que reflejan cuadros de insuficiencia respiratoria y complicaciones derivadas de mala función diafragmática con el fin de ilustrar la utilidad del uso de los ultrasonidos como método de diagnóstico de la disfunción diafragmática. 
Tradicionalmente, la evaluación de la función diafragmática se lleva a cabo a través de la historia, el examen físico, la prueba de aspiración fluoroscópica, los estudios de conducción nerviosa y la electromiografía (EMG). Los estudios de conducción nerviosa y EMG en este entorno son desafiantes, incómodos y pueden causar complicaciones graves, como neumotórax. Las pruebas de función pulmonar pueden ayudar a diagnosticar la debilidad del diafragma, pero su exactitud y reproducibilidad están limitadas por la dependencia del volumen pulmonar, el esfuerzo del paciente $y$ el amplio rango de variabilidad dentro del rango normal (2).

La ecografía neuromuscular es una técnica que ahora se utiliza para obtener imágenes del diafragma en condiciones normales y patológicas, dados los avances recientes que permiten imágenes de alta resolución. La ecografía es portátil, omnipresente en instalaciones médicas, no presenta riesgo de radiación ionizante y permite la visualización de estructuras debajo y encima del diafragma. Tiene la ventaja de evaluar los componentes estructurales y funcionales del diafragma en la cabecera del paciente. Se ha demostrado que la ecografía es similar en exactitud a la mayoría de las otras modalidades de imágenes para la evaluación del diafragma (1).

La técnica de evaluación de la función diafragmática mediante uso de la ecografía es sencilla con una curva de aprendizaje corta. En pacientes adultos, un estudio informó que la capacitación de los operadores de ultrasonido para identificar el diafragma y medir su grosor toma de tres a cinco sesiones que duran entre 10 y 15 minutos cada una $(3,4)$.

El espesor del diafragma se mide habitualmente en la zona de aposición del diafragma a la caja torácica; en la evaluación en modo $\mathrm{B}$, se accede a los hemidiafragmas a través de los espacios intercostales. Con el paciente en decúbito supino, se utiliza una sonda lineal multifrecuencia (7-18 $\mathrm{MHz})$ posicionada en el plano longitudinal en la línea axilar anterior, entre el $7^{\circ}$ y $8^{\circ} \mathrm{o}$ $8^{\circ}$ y $9^{\circ}$ espacios intercostales. Las evaluaciones se realizan en la zona de aposición, donde el diafragma se apoya en la caja torácica inferior (4) (Figura 2). El diafragma normal es visualizado entre dos líneas ecogénicas (Figura 3): la de la pleura parietal y la de la membrana peritoneal. Durante la inspiración, la musculatura de un diafragma sano se contrae y las fibras se acortan con aumento del volumen muscular y espesor consecuente. En la evaluación en modo $\mathrm{M}$, podemos obtener la fracción de acortamiento del diafragma definida como grosor inspiratorio - espesor espiratorio / espesor inspiratorio $\times 100$, que cuantifica el grado de engrosamiento muscular, siendo definida como disminuida cuando este resultado es menor del $30 \%(1,2,4)$.

Se ha encontrado que la fracción de acortamiento es un índice preciso de la carga de trabajo de los músculos respiratorios. Una revisión sistemática sobre la literatura actual que evalúa la utilidad y la precisión del uso de la ecografía para evaluación de función diafragmática en pacientes de unidades de cuidados intensivos incluyó veinte estudios. La ecografía se realizó con diferentes técnicas para medir la excursión inspiratoria diafragmática, el espesor del diafragma y la fracción de acortamiento. Los límites óptimos que incluyen los estudios de este metaanálisis variaron de 10 a $14 \mathrm{~mm}$ para la excursión y $30-36 \%$ para la fracción de acortamiento. Concluye que el manejo de la ecografía con esta finalidad es factible, altamente reproducible y permite detectar la 
disfunción diafragmática en el paciente crítico

El uso de la ecografía torácica no solo ha demostrado superioridad en el diagnóstico diferencial de la disnea y la insuficiencia respiratoria de origen neuromuscular frente a las técnicas convencionales sino que además ha supuesto un cambio de actitud terapéutica al poder incorporar su uso para optimizar el manejo del destete de la ventilación mecánica al ingreso del paciente en las unidades de cuidados intensivos (5). Varias publicaciones apoyan el hecho de que en el destete de la ventilación mecánica y las pruebas de respiración espontánea, tanto la excursión diafragmática como las mediciones de engrosamiento diafragmático se han utilizado para predecir el éxito o el fracaso de la extubación $(4,6,7)$.

En general, la ecografía es una modalidad de diagnóstico clínicamente valiosa pero tiene potenciales limitaciones. La imagen por ultrasonido ha sido criticada tradicionalmente por ser observador dependiente. Además determinadas consideraciones

anatómicas deben tenerse en cuenta como que la excursión hacia abajo del pulmón y la ventana más pequeña del bazo a la izquierda son dos posibles impedimentos para la visualización exitosa de ese hemidiafragma. Un escollo encontrado en la ecografía en pacientes con grandes derrames pleurales es la presencia de un movimiento diafragmático paradójico cuando los pacientes se examinan en posición de pie, lo que típicamente sugiere parálisis. Se ha informado que este hallazgo vuelve al movimiento normal cuando se realiza una ecografía con el paciente en decúbito supino y por lo tanto se recomienda esa posición. También se han notificado movimientos paradójicos de un diafragma no paralizado en el hidrotórax, neumotórax a presión negativa, pulmón fibrosis, atelectasia y absceso subfrénico $(7,8)$. Otra limitación del uso generalizado de la ecografía para la evaluación del diafragma es la falta de valores de referencia para los parámetros del diafragma en pacientes con enfermedad pulmonar o neuromuscular, porque hay diferentes rangos de volúmenes pulmonares para la respiración tranquila, la respiración profunda o las maniobras de inspiración. Según la posición corporal, el peso, la altura, la enfermedad pulmonar subyacente, o la condición física del sujeto, se ha demostrado que los músculos superiores de la caja torácica y del cuello contribuyen en mayor medida al volumen inspirado en ciertos sujetos (8). Por último reseñamos que los parámetros de ultrasonido del espesor y la excursión también pueden variar dependiendo del punto de medición inicial que escogemos como fin de expiración o comienzo de la inspiración.

\section{Conclusiones}

La ecografía del diafragma tiene su aplicación principalmente en pacientes en cuidados intensivos. En tales pacientes la evaluación del diafragma por ultrasonidos se puede utilizar para hacer un diagnóstico diferencial rápido de la disnea, predecir con éxito destete de la ventilación mecánica y adecuar las decisiones con respecto a ajustes en los parámetros de ventilación mecánica, controlar la carga de trabajo respiratorio y para investigar la debilidad o parálisis diafragmática asociada a determinadas cirugías y lesiones traumáticas.

Se necesitan más estudios que establezcan valores de referencia para el espesor del diafragma, la amplitud de la excursión y la velocidad que tienen en cuenta la fase del ciclo respiratorio. En conclusión, la ecografía es una técnica prometedora para la evaluación de la estructura y la función dinámica del diafragma. Es preciso, reproducible 
y relativamente fácil de aprender. El hecho de que no requiera traslado o movilización del paciente para su realización es muy importante para pacientes críticamente enfermos con ventilación mecánica. En un futuro debería valorarse la ecografía como la modalidad de elección para la evaluación de la parálisis del diafragma, especialmente en pacientes neonatales, pediátricos y en estado crítico.

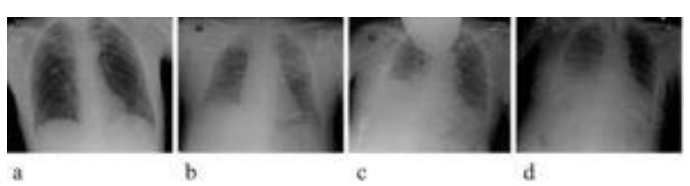

Figura 1: Radiografía de tórax de los casos clínicos. a. Caso 1: Parálisis diafragmática secundaria a lesión medular cervical. b. Caso 2: Aquinesia diafragmática por denervación traumática. c. Caso 3: Disfunción de marcapasos diafragmático. d. Caso 4: Paresia diafragmática unilateral tras hepatectomía derecha.

a

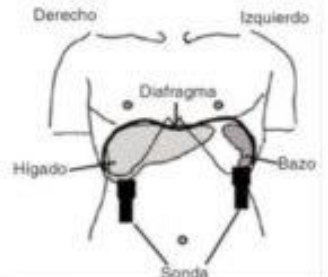

b

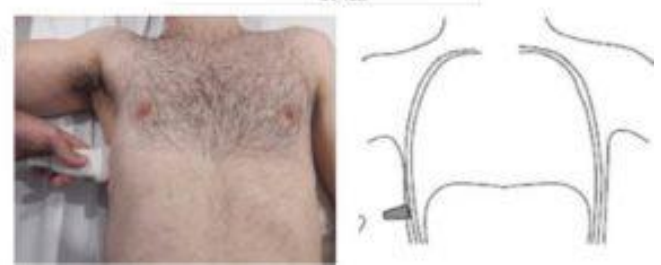

Figura 2: Técnica de evaluación ecográfica de la función diafragmática. a. Ventanas de insonación: hemidiafragma derecho e izquierdo, ventanas acústicas hígado y bazo. b. Colocación del transductor para la insonación diafragmática en plano longitudinal en la línea axilar anterior, entre el $7^{\circ}$ y $8^{\circ}$ espacios intercostales, en la zona de aposición del diafragma con la caja torácica.
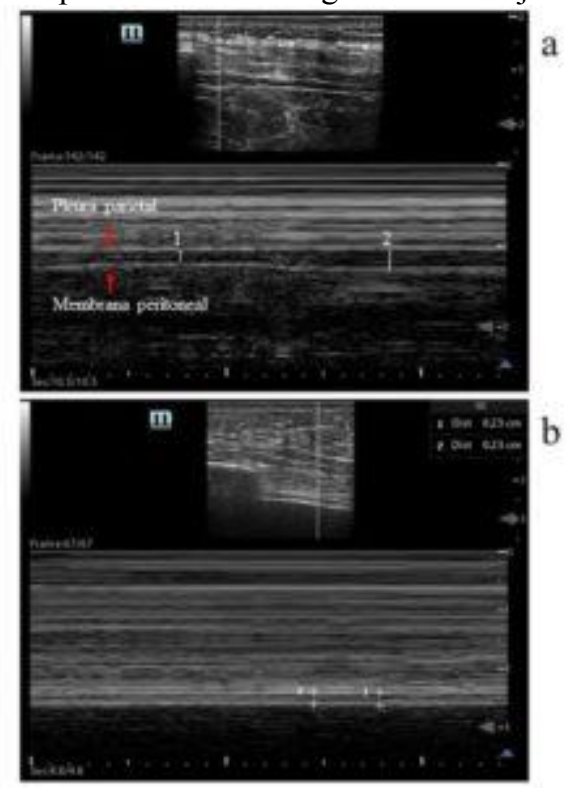

Figura 3: Modo M ecográfico para evaluación de contracción del diafragma. a. Ecografía con contracción diafragmática normal. b. Ecografía del caso 2. Aquinesia diafragmática por denervación traumática. Fracción de acortamiento del diafragma definida como grosor inspiratorio - espesor espiratorio / espesor inspiratorio $\times 100$. Resultado de la fracción de acortamiento en el caso 2 igual a 0 , aquinesia total.

\section{Bibliografía}

- Gerscovich EO, Cronan M, McGahan JP, Jain $\mathrm{K}$, Jones $\mathrm{CD}$, McDonald C. Ultrasonographic evaluation of diaphragmatic motion. $\mathrm{J}$ Ultrasound Med 2001; 20:597-604. (PubMed) ( $\underline{\text { HTML) }}$ ( $\underline{\text { PDF }})$

- Sarwal A, Walker FO, Cartwright MS. Neuromuscular ultrasound for evaluation of the diaphragm. Muscle Nerve. 2013 Mar;47(3):31929. ( PubMed) (

- DiNino E, Gartman EJ, Sethi JM, McCool FD. Diaphragm ultrasound as a predictor of successful extubation from mechanical ventilation. Thorax. 2014 May;69(5):423-7. ( $\underline{\text { PubMed }}$ ( $\underline{\text { HTML) }}$ (DF)

- Zambon M, Greco M, Bocchino S, Cabrini L, Beccaria PF, Zangrillo A. Assessment of diaphragmatic dysfunction in the critically ill patient with ultrasound: a systematic review. Intensive Care Med. 2017 Jan;43(1):29-38. (PubMed) 
- Serna Gandía M, Vicho Pereira R, Cuesta Montero P, Carpi Femenía I, Benlloch Beitia A. Ultrasonografía en UCI. Rev electron Boletín SCI 2016; Vol 2 (1): 1. (ㄴML)

- Matamis D, Soilemezi E, Tsagourias M, Akoumianaki E, Dimassi S, Boroli $\mathrm{F}$ et al. Sonographic evaluation of the diaphragm in critically ill patients. Technique and clinical applications. Intensive Care Med. 2013; 39:801-10. (PubMed)

- Alexander C. Diaphragm movements and the diagnosis of diaphragmatic paralysis. Clin Radiol 1966; 17:79-83. (ubMed)

-Wait JL, Nahormek PA, Yost WT, Rochester DP. Diaphragmatic thickness-lung volume relationship in vivo. J Appl Physiol 1989;67: 1560-1568. (PubMed)

Correspondencia al autor

Luisa María Charco Roca

luisacharco@gmail.com

Facultativo Especialista en Anestesiología y Reanimación.

Hospital General Universitario de Albacete

Aceptado para blog en agosto de 2018. 